\title{
A Simplified Technique for Fabrication and Characterization of a Custom Ocular Prosthesis using Light-cured Stains
}

\author{
${ }^{1}$ Sohil Rajkumar Daswani, ${ }^{2}$ Meena Ajay Aras, ${ }^{3}$ Vidya Chitre, ${ }^{4}$ Ashwin Mysore
}

\begin{abstract}
An ocular prosthesis which gives attention to the accurate duplication of natural color, contour, size and ocular orientation will provide realism and symmetry for patients who need it. This article describes a simplified technique of converting a stock eye shell into a well-fitting custom ocular prosthesis that requires minimal clinical procedures and simplified laboratory procedures. The color matching of the sclera of the prosthesis was performed using light-cured resin-based stains that are simple to use and give a more predictable and lifelike appearance when compared to the conventional acrylic 'paint on' technique.
\end{abstract}

Keywords: Custom ocular prosthesis, Resin stains, Scleral characterization, Maxillofacial prosthesis.

How to cite this article: Daswani SR, Aras MA, Chitre V, Mysore A. A Simplified Technique for Fabrication and Characterization of a Custom Ocular Prosthesis using Lightcured Stains. Int J Prosthodont Restor Dent 2015;5(1):17-20.

Source of support: Nil

Conflict of interest: None

\section{INTRODUCTION}

The loss of an eye is associated with significant emotional and psychological disturbance to the patient and, thus, warrants immediate management and rehabilitation by a specialist. In many cases, a stock eye prosthesis is used as a substitute for a natural eye. However, such a prosthesis may not perfectly adapt to the tissue bed of the eye and it usually causes significant discomfort and fails to satisfy the patients esthetic demands. A variety of techniques have been described for the fabrication of a custom ocular prosthesis, but they require numerous patient appointments and complex clinical and laboratory procedures. Previous techniques ${ }^{1-4}$ described for characterization of the sclera using acrylic paints and red rayon filaments are extremely technique sensitive and do not always result in a predictable outcome.

\footnotetext{
${ }^{1}$ Postgraduate, ${ }^{2}$ Professor and Head, ${ }^{3}$ Professor, ${ }^{4}$ Lecturer

${ }^{1-4}$ Department of Prosthodontics, Goa Dental College and Hospital, Goa, India
}

Corresponding Author: Sohil Rajkumar Daswani, Postgraduate Department of Prosthodontics, Goa Dental College and Hospital Goa, India, Phone: 9822015883, e-mail: sohildaswani@gmail.com
This article presents a simple yet effective method of converting a stock eye shell into a well-fitting, custom ocular prosthesis with characterization of the sclera requiring only two patient appointments.

\section{TECHNIQUE}

\section{First Appointment}

1. A stock eye shell was selected such that the dimensions and color of its iris-pupil complex closely resembled the contralateral eye (Fig. 1). The stock eye shell was trimmed to fit the eye socket and it served as a custom tray for making the ocular impression.

2. Autopolymerizing acrylic resin (Pattern Resin, GC Corp, Tokyo, Japan) was manipulated to form a stem that was attached to the center of the iris with sticky wax. This stem acted as a handle for the custom tray and an indicator for the orientation of the visual axis.

3. Low fusing compound (Pinnacle tracing sticks, Dental Products of India Ltd., Mumbai, India) was applied to the tissue surface of the prosthesis, tempered and inserted into the ocular defect and maintained in situ for 30 seconds. Low fusing compound was added and molded in the defect until the desired orientation of the visual axis was obtained.

4. Polyether light body impression material (Impregum Soft; 3M ESPE, Seefeld, Germany) was then injected on to the tissue surface of the prosthesis and inserted in the eye for accurate reproduction of surface details.

5. After about 5 minutes once the impression material set completely, it was retrieved from the defect (Fig. 2).

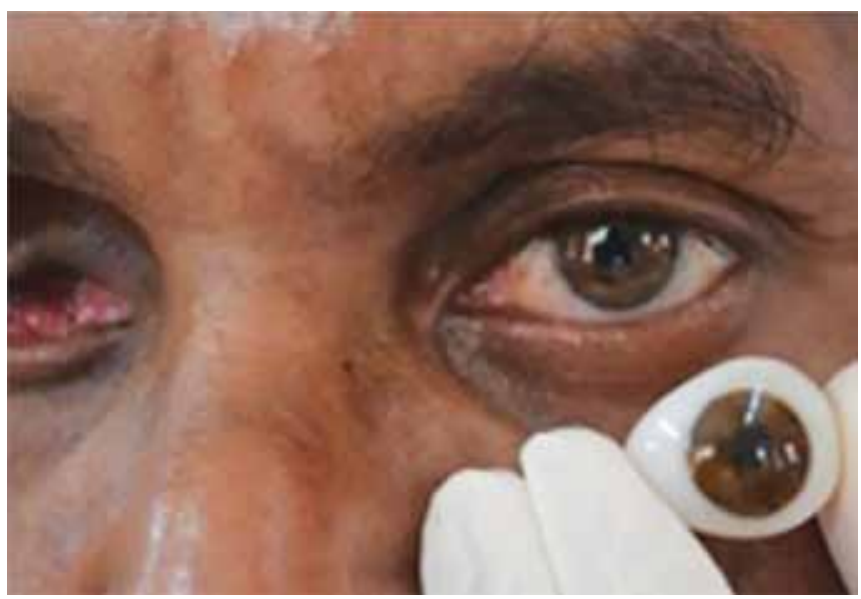

Fig. 1: Matching of the stock iris to the contralateral natural eye 
The position of the iris and the size of the palpebral openings were compared with those of the normal eye.

6. The impression surface was painted with type III dental stone (Kalstone, Kalabhai Pvt Ltd., Mumbai, India) and was placed in a flexible rubber cup filled with type III dental stone to get a cast. This cast was then invested in a flask filled with type II gypsum material (Kaldent, Kalabhai Pvt Ltd., Mumbai, India) (Fig. 3).

7. After completion of setting, the impression material was removed from the flask and replaced by molten wax (Modeling Wax, The Hindustan Dental Products, Hyderabad, India).

8. After the wax cooled, the flask was reopened and the stock eye shell along with the hardened wax on the tissue surface was retrieved (Fig. 4). This was tried-in the patient's eye and its positioning with respect to the adjacent eye was checked (Fig. 5). After the trial, wax on the tissue surface of the stock eye shell was removed and the shell was repositioned in the upper half of the flask.

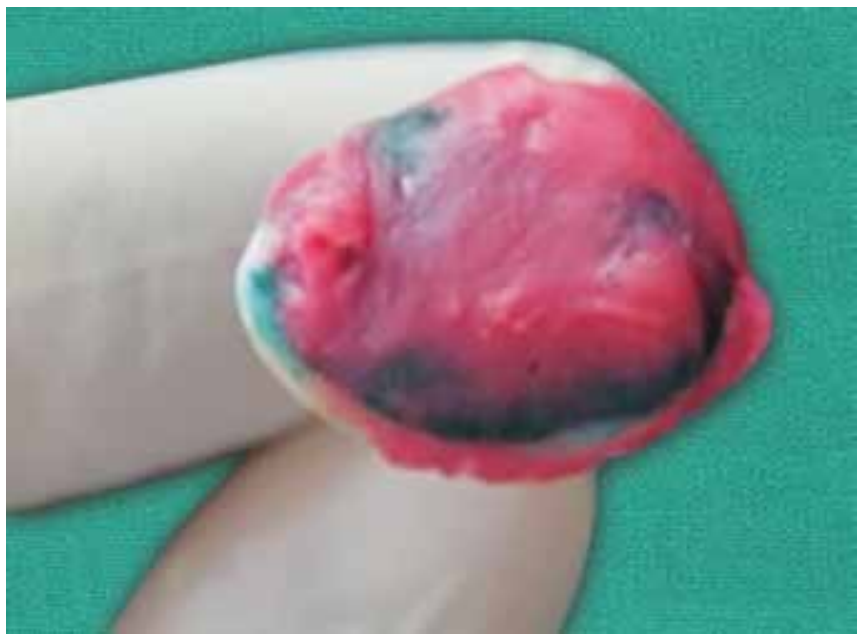

Fig. 2: Impression made using low fusing compound and polyether light body

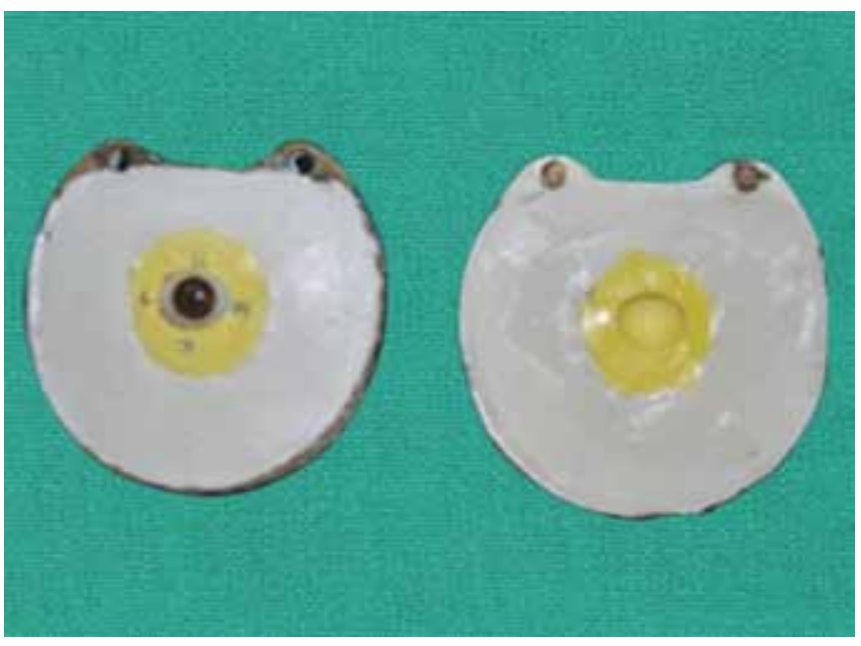

Fig. 3: Base and counter flasks after setting of dental plaster
9. Clear polymethyl methacrylate (Heat Cure, Dental Products of India Ltd., Mumbai, India) was mixed and packed in the mold space, and compression molding was carried out (Fig. 6). A short curing cycle was followed for the acrylization. After processing, the prosthesis was recovered from the flask, and finishing and polishing were done. The stock eye shell was converted to a custom ocular prosthesis (Fig. 7).

\section{Second Appointment}

10. This custom ocular prosthesis was tried-in the patient's eye to check for extent, fit comfort and iris position.

11. Characterization of the sclera was carried out using light curing stains (SR Adoro, Ivoclar Vivadent, Schaan, Liechtenstein) and the light-curing unit (Targis Power Upgrade, Ivoclar Vivadent, Leichtenstein). These stains contain urethane dimethacrylate (47-48 wt\%) and silicon dioxide (49-50 $\mathrm{wt} \%$ ) and are routinely used for characterization of acrylic denture teeth.

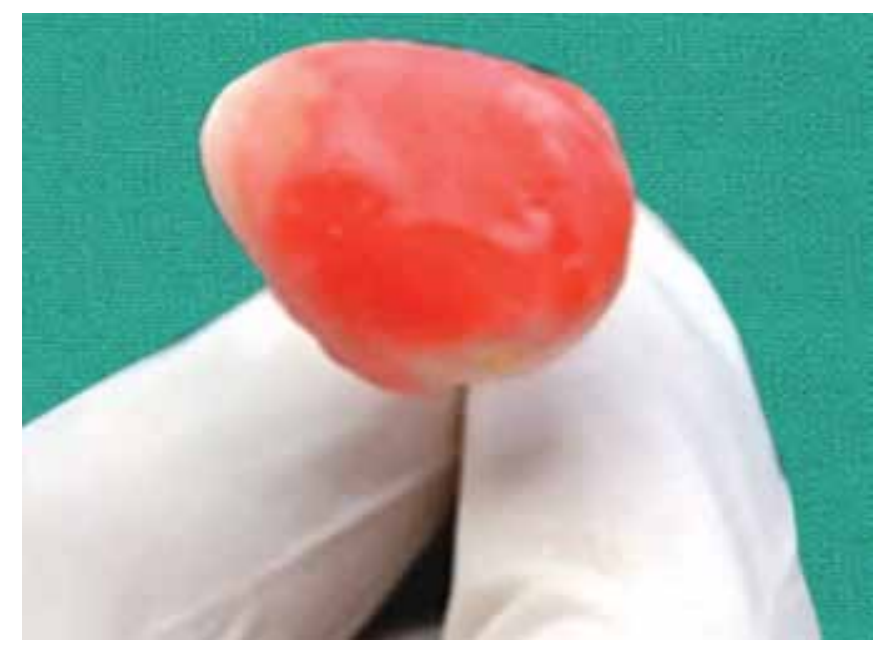

Fig. 4: Hardened wax on the tissue surface of the stock eye shell

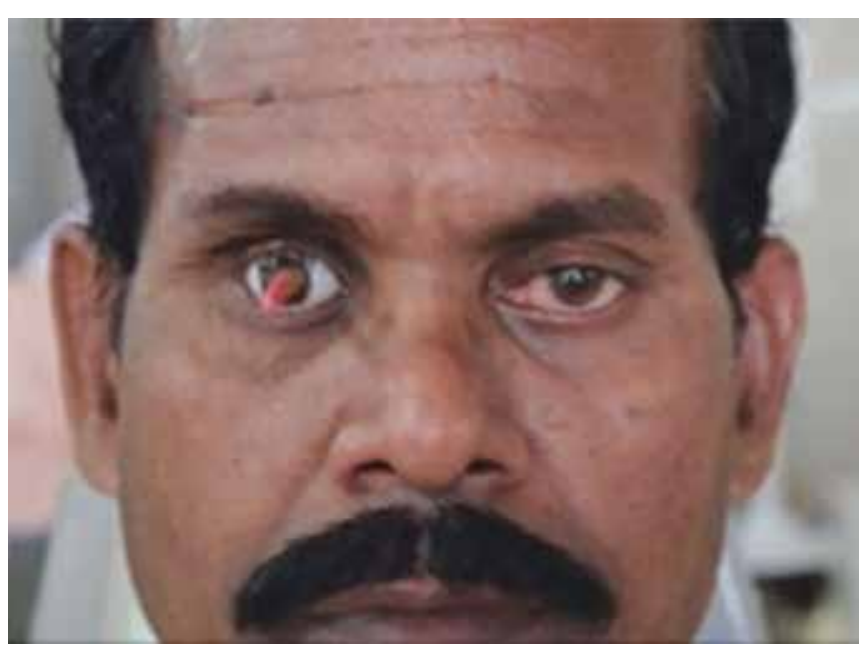

Fig. 5: Try-in and verification of the visual axis of orientation 


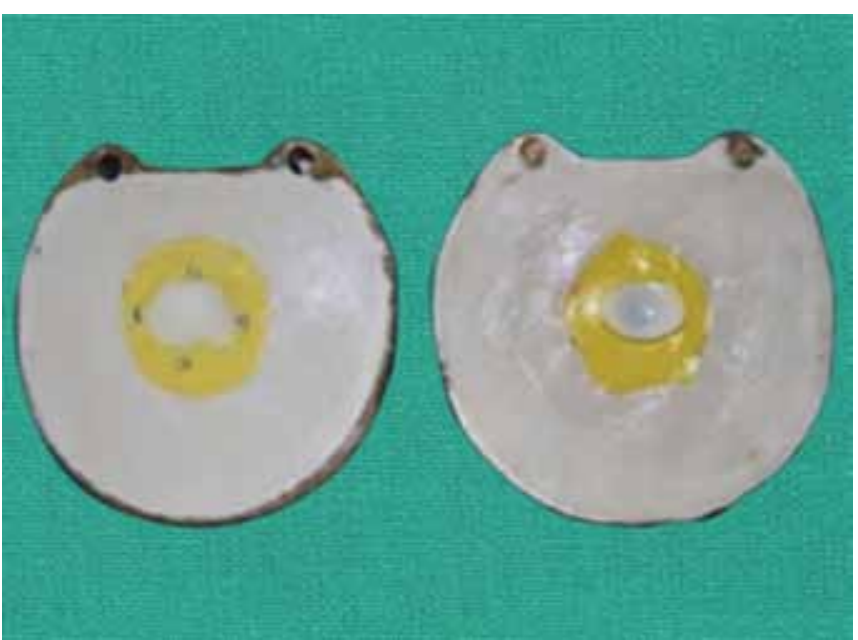

Fig. 6: Clear heat-cured acrylic resin packed into mold space

12. The scleral part of the prosthesis was painted with the light curing stains using a fine artist's brush to match the sclera of the contralateral eye (Fig. 8). According to manufacturer recommendations, the stains on the prosthesis were first photoactivated for 40 seconds (Quick curing unit, Ivoclar Vivadent, Leichtenstein). The prosthesis was then placed in the Targis Power Upgrade unit for 16 minutes under light and vacuum to complete the polymerization.

13. After cleaning the prosthesis with mild soap, the prosthesis was delivered to the patient (Fig. 9).

\section{DISCUSSION}

In this technique, the tissue surface of the stock eye shell was modified and acted as the final impression which helped in maintaining the position of the iris at the impression stage itself. This obviated the need for complex iris positioning techniques ${ }^{5-7}$.

The conventional techniques ${ }^{1-4}$ for characterization of the sclera using acrylic paints and red rayon filaments

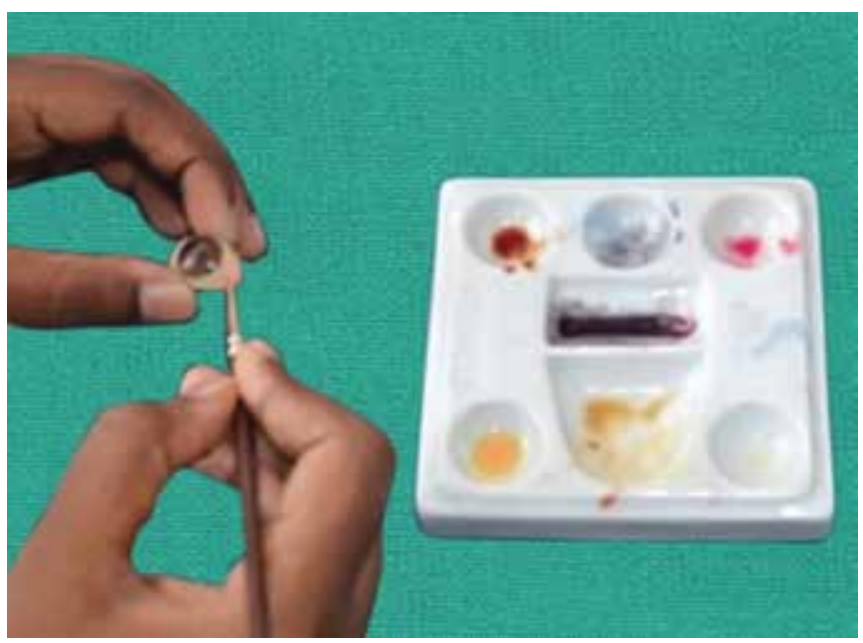

Fig. 8: Painting of the sclera with stains

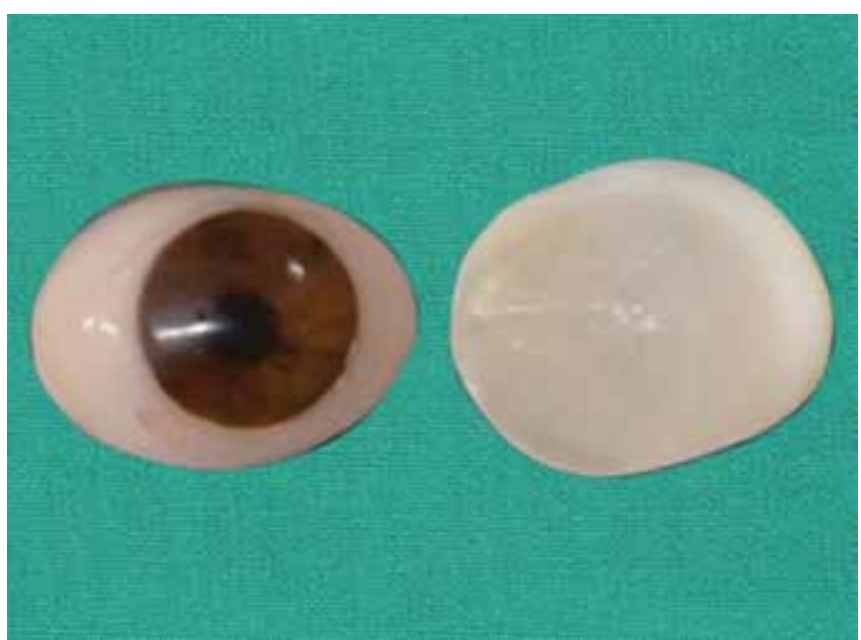

Fig. 7: Stock eye shell converted into a custom ocular prosthesis

requires an additional layer of heat cure acrylic resin to be processed over the paint as a protective covering. This often leads to smudging of the paint and a dull appearance of the final prosthesis. Also, the additional protective layer of acrylic required, after processing of the prosthesis, makes it difficult to predict the final contour of the prosthesis.

In this technique, the above problems have been overcome with the use of light-cured resin-based stains. The characterization of the sclera can be completed chairside as the stains have a short final curing time of 16 minutes. They do not require an additional protective layer after final curing, thus, the colors do not appear dull after curing and it does not alter the final contour of the prosthesis.

The primary limitation of this technique is the need for specialized equipment like the Targis Power Upgrade light-curing unit, which is uncommon in private clinics. Also, the long-term stability of these resin-based stains in the orbital environment is still to be evaluated.
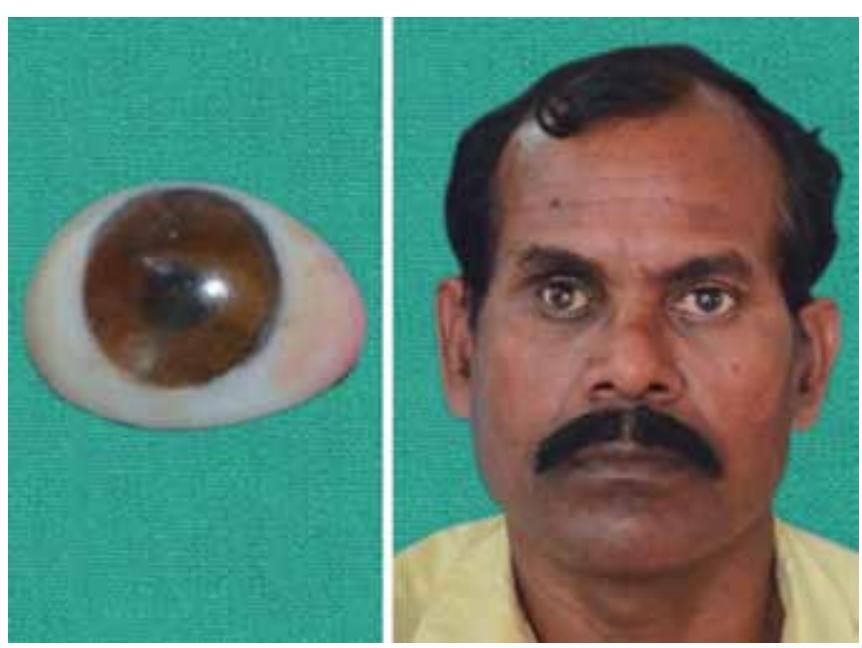

Fig. 9: Final prosthesis 


\section{CONCLUSION}

This article describes a simplified technique of converting a stock eye shell into a well-fitting custom ocular prosthesis that requires minimal clinical procedures and simplified laboratory procedures. The color matching of the sclera of the prosthesis using light-cured resin-based stains is simpler, quicker and gives a more lifelike appearance when compared to the conventional acrylic 'paint on' technique.

\section{REFERENCES}

1. Brown KE. Fabrication of an ocular prosthesis. J Prosthet Dent 1970 Aug;24(2):225-235.
2. Cain JR. Custom ocular prosthetics. J Prosthet Dent 1982 Dec;48(6):690-694.

3. Taicher S, Steinberg HM, Tubiana I, et al. Modified stock eye ocular prosthesis. J Prosthet Dent 1985 Jul;54(1):95-98.

4. Firtell DN, Anderson CR, Donnan ML. Vein application technique for ocular prostheses. J Prosthet Dent 1975 Aug;34(2):192-194.

5. McArthur DR. Aids for positioning prosthetic eyes in orbital prostheses. J Prosthet Dent 1977;37(3):320-326.

6. Roberts AC. An instrument to achieve pupil alignment in eye prosthesis. J Prosthet Dent 1969;22(4):487-489.

7. Guttal SS, Patil NP. A simple method of positioning the iris disk on a custom-made ocular prosthesis: a clinical report. J Prosthodont 2008 Apr;17(3):223-227. 